\title{
Single port laparoscopic hysterectomy: Feasibility and safety
}

\author{
Soon Pyo Lee, Sun Hye Yang, Chun Hoe Ku, Sung Ho Lee, Jin Woo Shin*
}

Department of Obstetrics and Gynecology, Gil Medical Center, Gachon University, Incheon, South Korea

Email: *jwshin@gilhospital.com

Received 24 June 2013; revised 26 July 2013; accepted 6 August 2013

Copyright (c) 2013 Soon Pyo Lee et al. This is an open access article distributed under the Creative Commons Attribution License, which permits unrestricted use, distribution, and reproduction in any medium, provided the original work is properly cited.

\begin{abstract}
Objective: To evaluate the feasibility and safety of single-port laparoscopic hysterectomy comparing with multi-port laparoscopic hysterectomy in treatment of benign uterine diseases. Methods: Data were collected retrospectively by review of the medical records of 252 patients who underwent multi-port or single-port laparoscopic surgery for treatment of benign gynecologic diseases. Laparoscopy assisted vaginal hysterectomy (LAVH) was performed for single-port surgery and LAVH and total laparoscopic hysterectomy (TLH) were performed for multi-port surgery. Demographic variables were collected and analyzed by independent $t$-test and Pearson Chi-Square test. The primary outcome was analyzed by independent $t$-test and Fisher's Exact test. Results: A longer operative time was observed in the multi-port surgery group compared with that of the single-port group $(p<0.05)$. No difference with respect to change of Hemoglobin between the preoperative level and that of the postoperative first day, the number of days from the operation to discharge, uterine weight, and the rate of laparotomy conversion and complications were observed between the two groups. Conclusion: Single-port laparoscopic hysterectomy for treatment of benign uterine diseases is a safe and feasible method.
\end{abstract}

Keywords: Laparoscopic Hysterectomy; Benign Uterine Diseases; Single-Port

\section{INTRODUCTION}

Laparoscopic surgeries have been widely reported to offer benefits, such as shorter hospital stays, more rapid recoveries, less post operative pain, lower complication rates, cost-effectiveness, and patient preference [1,2]. Ef-

\footnotetext{
"Corresponding author.
}

forts to decrease the numbers and size of wounds were highlighted to date and the recent approach has been single-port laparoscopic surgery [3-5]. This study was conducted in order to evaluate the feasibility and safety of single-port laparoscopic hysterectomy compared with multi-port laparoscopic hysterectomy in treatment of benign uterine diseases.

\section{MATERIALS AND METHODS}

We conduct a retrospective review of the medical records of 143 patients who underwent single port laparoscopic surgery in the Department of Obstetrics and Gynecology of a university hospital in Korea between Aug. 2011 and Jan. 2013. Data on multiport surgery was quoted extensively in the previously published paper [6]. The hospital Institutional Review Board approved the study. Cases of total hysterectomy were included in the study. Cases of adnexa surgery without hysterectomy were excluded from the study. Women with malignancies and planned abdominal or vaginal surgeries were excluded. The same surgeon performed all multi-port and single-port laparoscopic surgeries during the study period.

After induction of general anesthesia, the patients were placed in the dorsal lithotomy position with the buttocks well off the table, prepped, and draped. The RUMI system (Cooper Surgical, Trumbull, CT, USA) was used in the performance of total laparoscopic hysterectomy (TLH) and a reusable rigid uterine manipulator was used in the performance of laparoscopy-assisted vaginal hysterectomy (LAVH).

In cases of multi-port laparoscopic hysterectomy, a 10 $\mathrm{mm}$ transverse incision was made just above or below the umbilicus for the Verres needle and the primary trocar. After insufflation of $\mathrm{CO}_{2}$ up to a pressure of 15 $\mathrm{mmHg}$, a $10 \mathrm{~mm}$ trocar was placed and a $0^{\circ}$ telescope with a camera was inserted. Three additional ancillary trocars (5 mm trocars for the suprapubic and right midabdomen and a $10 \mathrm{~mm}$ trocar for the left mid-abdomen) 
were placed. All trocars, except the primary trocar, were inserted under direct vision of the telescope. The intraabdominal pressure was fixed at $12 \mathrm{mmHg}$ by the automated insufflator during the surgical procedure.

The hysterectomy through multiport laparoscopy was begun by dissection and hemostasis of the infundibulopelvic or ovarian ligament using an EndoGIA (Tyco Healthcare). The procedure formultiport laparoscopic hysterectomy was usual and has been described previously [6].

In cases of single-port laparoscopic hysterectomy, a 3 $\mathrm{cm}$ vertical incision was made on the umbilicus and fascia and peritoneum were opened using scissors under direct vision. The Octo-port ${ }^{\circledR}$ (Dalim, Korea) system was used as the gate of entry to the peritoneal cavity. Dissection and hemostasis of the infundibulopelvic or ovarian ligament and further dissection to the uterine artery were performed using an Enseal ${ }^{\circledR}$ vessel sealing device (Ethicon Endo-Surgery, Cincinnati, OH). The entire procedure for single port laparoscopy was performed by LAVH.

The operative time was calculated as the time that elapsed from scrubbing the surgical field to establishing closure of the abdomen. The change in hemoglobin was defined as the difference between the preoperative hemoglobin and the hemoglobin of the next day after surgery.

All continuous data were compared using the Independent $t$-test. Pearson Chi-square test and Fisher's Exact test were used for comparison of the difference between the TLH and LAVH groups. The Statistical Package for the Social Sciences for Windows, version 17.0 (SPSS, Inc., Chicago, IL, USA) was used to analyze all data. A $P$ value $<0.05$ was considered significant for all statistical tests.

\section{RESULTS}

Sixty two patients in single port surgery group, who were performed adnexa surgery only, were excluded from study. A total of 81 patients who underwent single-port and 171 patients who underwent multi-port laparoscopic total hysterectomy met the criteria for the study and were enrolled.

The age of subjects in the single-port surgery group was older than that of those in the multi-port surgery group. The number of previous abdominal surgeries was significantly higher in the multi-port surgery group compared with that of single-port. In addition, the body mass index, was also higher in the multi-port surgery group than that of single-port. Similar parity was observed between the two groups (Table 1). Longer operative time was observed in the multi-port surgery group compared with that of single-port. No difference with respect to change of Hemoglobin between the preoperative level and that of the postoperative first day, the number of days until discharge after the operation, uterine weight,
Table 1. Distributions of patients' characteristics.

\begin{tabular}{cccc}
\hline & Single port (\%) & Multi ports (\%) & $p$ \\
\hline No. of patients & 81 & 171 & \\
Age & $48.25 \pm 6.23$ & $45.31 \pm 6.30$ & $<0.05$ \\
Parity & $1.95 \pm 0.63$ & $1.96 \pm 0.92$ & 0.55 \\
No. abdominal surgery & & & \\
0 & $62(76.5)$ & $124(72.5)$ & \\
1 & $16(19.8)$ & $26(15.2)$ & \\
$>2$ & $3(3.7)$ & $27(15.8)$ & $<0.05$ \\
Body mass index & $22.90 \pm 2.47$ & $23.97 \pm 3.20$ & $<0.05$ \\
\hline
\end{tabular}

and the rate of laparotomic conversion and complications was observed between the two groups (Table 2).

This study included four cases of conversion to laparotomy, one $(1.23 \%)$ in the single-port surgery group and three $(1.75 \%)$ in the multi-port surgery group, which were caused by severe pelvic adhesion. Re-operation for bleeding control was performed in one case in the singleport surgery group and there was one case of ureteral injury and one case of bladder injury in the multi-port surgery group, however, these cases were managed properly with out occurrence of serious complications (Table 2). There was no occurrence of major vascular or bowel injuries.

\section{DISCUSSION}

The proportion of laparoscopic hysterectomies has been increasing as compared with hysterectomies performed through a laparotomy [7]. Recently, even more, a single port approach for malignant disease is upcoming and discussed all on the boil [3-5].

In a study comparing single-port surgery and multiport surgery, Behnia-Willison et al. reported that the single-port surgery improved cosmesis and reduced analgesic requirements [3], however others $[4,8]$ have reported opposite results. In this study, the authors did not conduct a scientific investigation of the cosmetic outcome and recovery profile such as post-operative pain score and cost, further study might discover these questions.

Longer operation time was observed in the multi-port surgery group, which is considered the patient's history of previous abdominal surgery, with a higher number in the multi-port surgery than in the single-port surgery group. The more abdominal operations performed, the more adhesions that develop, and the more time involved in adhesiolysis [6].

A variety of port systems are now available, such as a homemade port system [5], Covidien SILS port [3], and the Octoport system [9] used in the current study. The ideas for each system seem to be similar, however, the choice is up to the surgeon. 
Table 2. Primary outcomes.

\begin{tabular}{cccc}
\hline & Single port (\%) & Multi ports (\%) & $p$ \\
\hline Operation time (min) & $94.26 \pm 25.08$ & $112.59 \pm 33.68$ & $<0.05$ \\
$\begin{array}{c}\text { Hemoglobin change } \\
\text { (g/dL) }\end{array}$ & $1.61 \pm 1.33$ & $1.73 \pm 1.42$ & 0.52 \\
Post-operative stay (days) & $4.17 \pm 1.53$ & $3.71 \pm 2.09$ & 0.08 \\
Uterus weight (g) & $246.03 \pm 155.68$ & $223.98 \pm 131.77$ & 0.26 \\
Laparotomy conversion & $1(1.23)$ & $3(1.75)$ & 1.0 \\
Complications & $1(1.0)$ & $2(1.2)$ & 1.0 \\
\hline
\end{tabular}

The current study has a limitation, in that the data were quoted from the past study [6], however in spite of different periods, all surgeries were performed by one surgeon may overcome the bias due to time period and interpersonal variance. The learning period for singleport laparoscopic hysterectomy may be the first 20 cases, however, bias inexact comparison of the outcome of each surgery is still possible.

In the single-port surgery group, one case of re-operation for post-operative bleeding was reported. The patient was resent to the operative room after 5 hours of the initial operation and underwent surgery performed using a single-port approach. No active arterial bleeding was observed, however, generalized oozing was controlled by use of an electric device and the patient recovered without occurrence of serious complication.

The severe pelvic adhesion requires conversion of laparoscopic surgery to laparotomy, one case involving single-port surgery was converted to laparotomy, even use of ancillary trocars did not allow for maintenance of laparoscopic surgery.

TLH was reported as a significant risk factor for vaginal cuff dehiscence $[6,10]$. Extensive tissue destruction caused by thermal injury at the time of colpotomy using monopoloar scissors makes the vaginal cuff vulnerable to delayed healing and dehiscence. In order to avoid or lessen such avaginal cuff complication, topical injection of a vasoconstrictor at the colpotomy site followed by a sharp colpectomy using a laparoscopic scalpel should be considered [10]. In this study, vaginal cuff open and closure in single-port laparoscopic hysterectomy was performed like vaginal hysterectomy, so that there was no accident of cuff complication.

The single-port laparoscopic hysterectomy is safe and feasible method with acceptable complication. Study focusing on the concerns of cosmesis and post-operative pain should follow.

\section{REFERENCES}

[1] Lenihan Jr., J.P., Kovanda, C. and Cammarano, C. (2004) Comparison of laparoscopic-assisted vaginal hysterecto- my with traditional hysterectomy for cost-effectiveness to employers. American Journal of Obstetrics \& Gynecology, 190, 1714-1720. doi:10.1016/j.ajog.2004.02.059

[2] Muzii, L., Basile, S., Zupi, E., Marconi, D., Zullo, M.A. and Manci, N. (2007) Laparoscopic-assisted vaginal hysterectomy versus minilaparotomy hysterectomy: A prospective, randomized, multicenter study. Journal of $\mathrm{Mi}$ nimal Invasive Gynecology, 14, 610-615. doi:10.1016/j.jmig.2007.05.012

[3] Behnia-Willison, F., Foroughinia, L., Sina, M. and McChesney, P. (2012) Single incision laparoscopic surgery (SILS) in gynaecology: feasibility and operative outcomes. Australian and New Zealand Journal of Obstetrics and Gynaecology, 52, 366-370. doi:10.1111/j.1479-828X.2012.01443.x

[4] Mencaglia, L., Mereu, L., Carri, G., Arena, I., Khalifa, H. and Tateo, S. (2013) Single port entry-Are there any advantages? Best Practice \& Research Clinical Obstetrics \& Gynaecology, 18, 441-455. doi:10.1016/j.bpobgyn.2012.12.002

[5] Choi, Y.S., Shin, K.S., Choi, J., Park, J.N., Oh, Y.S. and Rhee, T.E. (2012) Single-port access laparoscopy-assisted vaginal hysterectomy: our initial experiences with 100 cases. Minimally Invasive Surgery, 2012, 1-5. doi:10.1155/2012/543627

[6] Shin, J.W., Lee, H.H., Lee, S.P. and Park, C.Y. (2011) Total laparoscopic hysterectomy and laparoscopy-assisted vaginal hysterectomy. Journal of the Society of Laparoendoscopic Surgeons, 15, 218-221. doi:10.4293/108680811X13071180406394

[7] David-Montefiore, E., Rouzier, R., Chapron, C. and Daraï, E. (2007) Surgical routes and complications of hysterictomy for benign disorders: A prospective observational study in French university hospitals. Human Reproduction, 22, 260-265. doi:10.1093/humrep/del336

[8] Yim, G.W., Lee, M., Nam, E.J., Kim, S., Kim, Y.T. and Kim, S.W. (2013) Is single-port access laparoscopy less painful than conventional laparoscopy for adnexal surgery? A comparison of postoperative pain and surgical outcomes. Surgical Innovation, 20, 46-54. doi:10.1177/1553350612439632

[9] Song, T., Kim, T.J., Kang, H.J., Choi, C.H., Lee, J.W. and Bae, D.S. (2011) Single-port access laparoscopic surgery using a novel laparoscopic port (Octo-Port). Taiwanese Journal of Obstetrics and Gynecoloy, 50, 436-440. doi:10.1016/j.tjog.2011.10.007

[10] Hur, H.C., Guido, R.S., Mansuria, S.M., Hacker, M.R., Sanfilippo, J.S. and Lee, T.T. (2007) Incidence and patient characteristics of vaginal cuff dehiscence after different modes of hysterectomies. Journal of Minimal Invasive Gynecology, 14, 311-317. doi:10.1016/j.jmig.2006.11.005 